\title{
Strategies to improve fiber utilization in swine
}

\author{
Brian J Kerr ${ }^{1 *}$ and Gerald C Shurson ${ }^{2}$
}

\begin{abstract}
Application of feed processing methods and use of exogenous feed additives in an effort to improve nutrient digestibility of plant-based feed ingredients for swine has been studied for decades. The following review will discuss several of these topics, including: fiber characterization, impact of dietary fiber on gastrointestinal physiology, energy, and nutrient digestibility, mechanical processing of feed on fiber and energy digestibility, and the use of exogenous enzymes in diets fed to growing pigs. Taken together, the diversity and concentration of chemical characteristics that exists among plant-based feed ingredients, as well as interactions among constituents within feed ingredients and diets, suggests that improvements in nutrient digestibility and pig performance from mechanical processing or adding exogenous enzymes to diets fed to swine depends on a better understanding of these characteristics, but also relating enzyme activity to targeted substrates. It may be that an enzyme must not only match a target substrate(s), but there may also need to be a 'cocktail' of enzymes to effectively breakdown the complex matrixes of fibrous carbohydrates, such that the negative impact of these compounds on nutrient digestibility or voluntary feed intake are alleviated. With the inverse relationship between fiber content and energy digestibility being well described for several feed ingredients, it is only logical that development of processing techniques or enzymes that degrade fiber, and thereby improve energy digestibility or voluntary feed intake, will be both metabolically and economically beneficial to pork production.
\end{abstract}

Keywords: Energy, Enzymes, Fiber, Growing-finishing pigs, Nutrient digestibility, Processing

\section{Introduction}

Plant carbohydrates can be classified into three categories: 1) simple sugars and their conjugates (glucose, fructose, etc.); 2) storage reserve compounds (starch); and 3) structural carbohydrates (cellulose, hemicellulose, etc.). Simple sugars and storage compounds are primarily digested in the upper gastrointestinal tract of pigs, although not completely, while structural carbohydrates are only partially degraded by the microflora in the cecum and large intestine [1]. Because most of the starch is removed from corn for ethanol and 'sugar' production and from wheat for flour production, resultant co-products (dried distillers grains with solubles-DDGS, corn gluten feed, and wheat middlings, respectively) contain concentrated levels of protein, minerals, and fiber [2]. With pigs being able to utilize moderate, but not high levels of fiber in the nursery [3,4] and finisher [5] phases of growth, there is a need to increase the ability of the pig to utilize the energy

\footnotetext{
* Correspondence: brian.kerr@ars.usda.gov

${ }^{1}$ USDA-ARS-National Laboratory for Agriculture and the Environment, Ames, IA 50011, USA

Full list of author information is available at the end of the article
}

associated with the structural carbohydrates contained in various 'high-fiber' co-products [6]. Due to record high feed prices around the world, it is essential that we find effective ways to minimize the cost associated with meeting the dietary energy and amino acid needs of all livestock and poultry, including swine. In order to accomplish this goal, we need to develop and evaluate technologies that increase digestibility of energy and other nutrients in grain co-products. Use of various processing techniques and exogenous enzymes are two technologies that offer promise for improving the nutritional value of high fiber co-products.

\section{"Fiber" in Swine Nutrition \\ Definition}

Unfortunately, "fiber" is perhaps the most poorly understood constituent of swine diets, and is generally described as a complex and highly variable component of plant-based feedstuffs (Figure 1) [7]. It is important to note that the analytical methods used to characterize "fiber" often overlap or may exclude fractions of other distinctly different carbohydrate fractions in a
C Biomed Central

(c) 2013 Kerr and Shurson; licensee BioMed Central Ltd. This is an Open Access article distributed under the terms of the Creative Commons Attribution License (http://creativecommons.org/licenses/by/2.0), which permits unrestricted use, distribution, and reproduction in any medium, provided the original work is properly cited. 


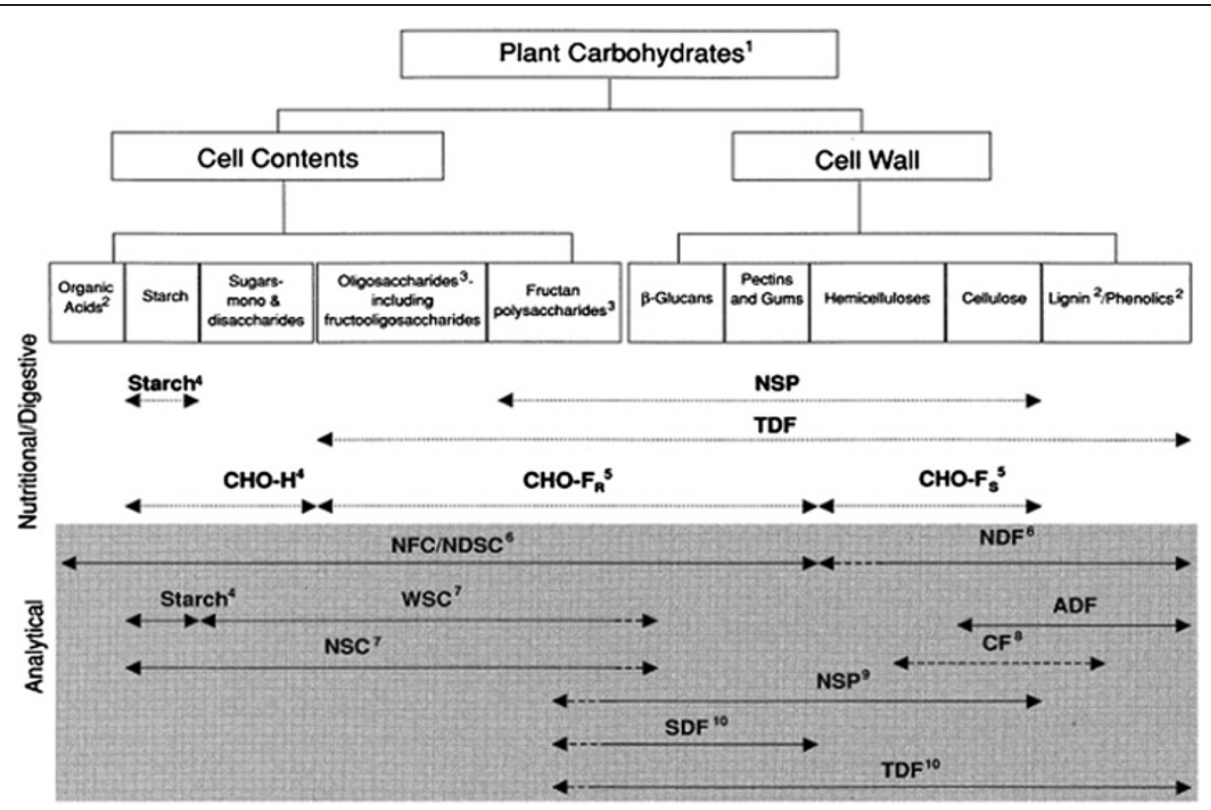

Figure 1 Nutritional and analytical classifications used to characterize plant carbohydrates [7].

feedstuff, and consequently, our ability to adequately relate analytical measures to fiber utilization has been problematic. Some fiber types are more digestible than others, and although they cannot be broken down by mammalian enzymes, they can be fermented by bacteria in the hindgut [8]. These fiber types are often termed "nonstarch polysaccharides" (NSP), where up to $90 \%$ of the cell walls of plants are made up of NSP; of which, cellulose, hemicellulose, and pectins are most abundant [9]. Other less abundant NSP include fructans, glucomannans, galactomannans, mucilages, $\beta$-glucans, and gums. Cellulose is found in tightly bound aggregates in plants, while hemicellulose and pectins have sugar side chains that allow them to be more readily broken down. Lignin is not a polysaccharide, but is a high molecular weight polymer, and is not considered a functional dietary constituent because it is indigestible by swine [8]. As shown in Figure 1, common analytical methods used to measure complex carbohydrates in high fiber feed ingredients and feeds include: crude fiber, acid detergent fiber (ADF), neutral detergent fiber (NDF), soluble and insoluble fractions of total dietary fiber (TDF), and NSP. Since each of these fiber methods measures several and sometimes different fractions of complex carbohydrates, they do not adequately relate to the energy value of feeds for swine.

\section{Energy value of fiber}

The digestibility of "fiber" in swine diets can vary dramatically from 0 to $97 \%$ depending upon the source of fiber [10], processing method [11], and concentration in the diet $[12,13]$. However, many NSP are partially fermentable in the hindgut and can be used to produce volatile fatty acids (VFA) such as acetate, propionate, and butyrate. These VFA are rapidly absorbed and have been shown to supply between 5 and $28 \%$ of the maintenance energy requirement of pigs [14-19]. However, the loss of energy due to methane, hydrogen, and fermentation heat decrease the amount of energy available to the pig from fermentation of fiber in the hindgut [8], thereby decreasing the efficiency of energy utilization $[20,21]$.

\section{Fiber alters the gastrointestinal tract}

1. Weight

Feeding high fiber diets results in a general increase in the total empty weight of the gastrointestinal tract $[12,16,22]$ and increased gastrointestinal secretions [8]. Jørgensen [23] showed that growingfinishing pigs fed diets containing high dietary fiber (NSP + lignin) (268 g/kg dry matter, DM) as compared to pigs fed diets low in dietary fiber $[59 \mathrm{~g} / \mathrm{kg} \mathrm{DM}$ ), had a significantly heavier stomach, cecum, and colon weights, as well as a longer colon.

2. Enterocyte proliferation Intestinal epithelial cell proliferation rate is stimulated by feeding high NSP diets $[24,25]$ leading to an increase in cell turnover rate. Growing pigs fed diets containing 10\% wheat straw had a $33 \%$ increase in the rate of jejunal and colonic cell proliferation, and a $65 \%$ increase in cells undergoing cell death [24].

3. Endogenous fluid secretion 
The secretion of endogenous fluids is also increased when feeding high fiber diets to pigs [26]. Secretions of saliva, gastric juice, and pancreatic juice were doubled when dietary fiber content was increased from 50 to $180 \mathrm{~g} / \mathrm{kg}$ in $50 \mathrm{~kg}$ pigs [27].

4. Maintenance energy requirement With the many changes in the characteristics of the gastrointestinal tract due to feeding a high fiber diet, the maintenance energy requirements of pigs may be increased by the extra metabolic demand due the nutrient needs for visceral organ development and maintenance $[8,26]$. Consequently, methods to improve fiber digestion would reduce these negative effects of fiber on animal metabolism.

5. Gastric emptying and satiety The rate of gastric emptying may decrease with the addition of certain forms of NSP. Guar gum and pectin increase the viscosity of the digesta [8] and water retention [28]. Growing pigs fed a high energy (starch, casein, soybean oil, and tallow) diet supplemented with 40 to $60 \mathrm{~g} / \mathrm{kg}$ guar gum had a reduced rate of gastric emptying of $33 \%$ to $52 \%$ after feeding, and a $27 \%$ reduction in DM concentration of the digesta [29,30]. High fiber diets may also contribute to earlier satiety resulting from gastric signals due to the elongation of the stomach wall. Feeding an increased amount of dietary fiber may lead to increased volume of digesta in the stomach, decreased transit time, and increased satiety. This is important in gestating sows because if they are satisfied physically and nutritionally, they appeared to be less stressed and exhibited decreased physical activity [31].

6. Digesta passage rate and nutrient utilization The passage rate of digesta can also be affected by feeding diets high in fiber. Some studies have shown increasing daily DM flow at the terminal ileum when NDF levels were increased in the diet [32]. Others have also shown up to a $14 \%$ and $23 \%$ increase in rate of passage when 75 to $300 \mathrm{~g}$ of bran or oatmeal co-products, respectively, was added to the diet [33]. These results suggest that the differences in rate of passage through the total digestive tract may be due to differences in the rate of passage through the large intestine, because neither fiber source had a significant effect on gastric emptying or passage through the small intestine [33]. Additionally, particle size of a fiber source may also contribute to the rate of passage. Bardon and Fioramonti [34] showed that a large particle size of wheat bran decreases transit time compared to a smaller particle size.
The amount of time the digestive contents spend in the large intestine can also affect the capacity for fermentation. Fiber fermentation in the cecum and colon results in the production of VFA (mainly acetic, propionic, and butyric acids) which are viable sources of energy. However, the energy density and digestibility of the diet usually decreases with the addition of NSP [8]. In addition, NSP reduces lipid absorption due to a partial inhibition of both lipolysis and intestinal fat absorption [35]. Nonstarch polysaccharides also decrease dietary nitrogen $(\mathrm{N})$ retention due to increased secretion of endogenous $\mathrm{N}$, which leads to increased bacterial $\mathrm{N}$ excretion [8]. Although minerals do not contribute energy directly to the diet, an impact of NSP on mineral utilization should also be considered (i.e., deficiencies or excesses could lead to physiological conditions that may ultimately affect energy absorption). However, the impact of NSP sources on mineral utilization appears to be minimal $[8,36]$.

\section{Mechanical Processing Effects on Fiber Utilization}

Limited data are available relative to the effect of processing (mechanical or chemical) on changes in fiber utilization in non-ruminants. Teitge et al. [37] reported that pelleting and micronizing, but not steam-flaking, resulted in a greater response to a dietary pentosanase in broilers fed diets containing rye, while Brenes et al. [38] indicated that autoclaving lupins had no impact on chick performance. Autoclaving high-tannin peas, in contrast to low-tannin peas, improved apparent metabolizable energy and apparent protein digestibility in Leghorn chicks [39]. In $80 \mathrm{~kg}$ pigs fed barley-based diets, pelleting had no effect on ileal or fecal apparent digestibilities of DM, gross energy (GE), crude protein $(\mathbf{C P})$, fat, or fiber (NSP + lignin), although it did increase pre-ileal apparent digestibility of starch [40]. In contrast to Teitge et al. [37], Graham et al. [40] reported that pelleting did not improve the digestibility response found when dietary ß-glucanase was added to the diet.

Poel et al. [41] reported that steam processing of faba bean cotyledons did not improve ileal digestibility of $\mathrm{CP}$, either due to the low level of trypsin inhibitor activity present in faba beans, or due to the trypsin inhibitor being sensitive to heat above the $100^{\circ} \mathrm{C}$ which was used in this study. Likewise, Thacker and Campbell [42] and Nyachoti et al. [43] showed little effect of micronization on nutrient digestibility coefficients. Pelleting of diets containing high levels of corn fiber (corn gluten feed), improved $\mathrm{N}$ balance, apparently due to the increased availability of tryptophan [44]. Extrusion is a heat processing method for feed ingredients that is commonly used in the commercial feed industry. However, very little is known about the effects of extruding corn and corn co-products on 
nutritional value [6]. Additional detailed information regarding the impact of feed processing on energy and nutrient digestibility has been published in reviews by Hancock and Behnke [45] and Stark [46].

\section{Effects of Exogenous Enzymes on Fiber Utilization Poultry vs. swine diets}

The addition of exogenous enzymes to animal feeds in efforts to improve nutrient digestion is not a new concept and responses have been reviewed in detail $[47,48]$. The majority of commercial enzyme products has been targeted toward poultry $[49,50]$ and are typically added to diets containing barley, oats, peas, rye, or wheat [51-54]. Few studies evaluating enzyme use in corn-soybean meal diets have been published [55].

\section{Enzymes in non-corn based swine diets}

As with poultry, the majority of research on adding enzymes to swine diets has focused on non-corn-based diets. Adding a multi-enzyme complex to diets containing barley and wheat has been shown to improve soluble NSP digestibility in $10 \mathrm{~kg}$ pigs, although growth performance was not affected [56]. Similarly, variation in responses from enzyme addition in pig diets has also been reported by Nonn et al. [57], who found no effect of enzyme supplementation on pig growth performance, even though they observed increased digestibility of crude fiber and cellulose. Likewise, Thacker and Campbell [43] indicated that although enzyme supplementation increased nutrient digestibility coefficients, there was little effect on pig growth performance. In contrast, Omogbenigun et al. [58] supplemented an enzyme cocktail (cellulase, galactanase, mannase, and pectinase) to a wheat-based diet fed in $6 \mathrm{~kg}$ pigs and observed an improvement in growth performance (growth rate and feed efficiency) over a $38 \mathrm{~d}$ period. Improved nutrient digestibility has also been reported by Yin et al. [59] who added xylanase to diets containing wheat co-products fed to $15 \mathrm{~kg}$ pigs and reported improved ileal and total tract apparent digestibility of DM, CP, and energy, especially in diets containing high levels of insoluble NSP. Lastly, adding an enzyme cocktail (fermentation extracts and solubles from $A$. niger and T. longibranchautum) to a diet containing $20 \%$ soy hulls improved DM and energy digestibility, but not $\mathrm{N}$ digestibility, in 33 to $51 \mathrm{~kg}$ pigs [60]. With soybean hulls having a large proportion of cellulose relative to other NSP, these data provide some evidence that cellulose digestion can be impacted in addition to hemicellulose and the more soluble forms of fiber.

\section{Enzymes in corn-based swine diets}

Limited research has been reported on the impact of exogenous enzymes on nutrient digestibility or pig performance when fed corn-based diets. Supplementation of $\beta$-glucanase to a corn-soybean meal-based diet had no impact on DM, energy, or CP digestibility in $6 \mathrm{~kg}$ pigs [61]. Likewise, supplementation of $\beta$-mannanase ( $\beta$-mannose is a part of hemicellulose) to a corn-soybean meal-based diet failed to show any effect on DM, energy, or $\mathrm{N}$ digestibility in $93 \mathrm{~kg}$ barrows [62]. However, adding $\beta$-mannanase improved feed efficiency in $6 \mathrm{~kg}$ pigs (42 d feeding period) and $14 \mathrm{~kg}$ pigs (21 d feeding period), and improved gain and feed efficiency, but had no impact on carcass composition, when fed from 23 to $110 \mathrm{~kg}$ [62]. Kim et al. [63] utilized a carbohydrase enzyme mixture $(\alpha-1,6$-galactosidase and $\beta-1,4$ mannanase) in corn-soybean meal-based diets fed to nursery pigs and reported an improvement in feed efficiency in two trials ( $35 \mathrm{~d}$ trial, 6.3 to $19.1 \mathrm{~kg} \mathrm{BW}$; and a $21 \mathrm{~d}$ trial, 8.0 to $15.2 \mathrm{~kg} \mathrm{BW}$ ), as well as an improvement in ileal energy digestibility. Supplementation of the carbohydrase enzyme mixture also decreased the concentration of stachyose in the proximal and distal small intestine, and raffinose concentration in the distal small intestine, suggesting that

Table 1 Effect of enzyme supplementation on growth performance, percent apparent ileal digestibility (AID), and total-tract digestibility (TTD) of nutrients in 7 kg pigs ${ }^{1}$

\begin{tabular}{|c|c|c|c|c|c|c|}
\hline \multirow[b]{2}{*}{ Performance } & \multicolumn{4}{|c|}{ Diet $^{2}$} & \multicolumn{2}{|c|}{ Statistics } \\
\hline & Control & $\begin{array}{c}C+E n z \\
A \\
\end{array}$ & $\begin{array}{c}C+E n z \\
B \\
\end{array}$ & $\begin{array}{c}C+E n z \\
C \\
\end{array}$ & SEM & P-value \\
\hline$\overline{A D G}, \mathrm{~g}$ & $224^{b}$ & $252^{a}$ & $263^{a}$ & $249^{a}$ & 7.9 & 0.02 \\
\hline ADFI, g & 432 & 435 & 456 & 414 & 17.8 & 0.42 \\
\hline G:F & $0.52^{b}$ & $0.58^{\mathrm{a}}$ & $0.58^{\mathrm{a}}$ & $0.61^{\mathrm{a}}$ & 0.02 & 0.01 \\
\hline \multicolumn{7}{|l|}{ AID, $\%$} \\
\hline DM & $60.1^{\mathrm{b}}$ & 65.8 & $66.1^{\mathrm{a}}$ & $66.7^{\mathrm{a}}$ & 1.5 & 0.01 \\
\hline Starch & $86.7^{b}$ & $92.6^{a}$ & $94.6^{\mathrm{a}}$ & $95.3^{\mathrm{a}}$ & 1.1 & 0.02 \\
\hline GE & $62.8^{b}$ & $70.0^{\mathrm{a}}$ & $69.7^{\mathrm{a}}$ & $71.4^{\mathrm{a}}$ & 0.9 & 0.01 \\
\hline $\mathrm{CP}$ & $62.1^{b}$ & $71.5^{\mathrm{a}}$ & $71.4^{\mathrm{a}}$ & $73.2^{\mathrm{a}}$ & 1.5 & 0.01 \\
\hline Phytate & $59.2^{\mathrm{b}}$ & $71.7^{\mathrm{a}}$ & $69.1^{\mathrm{a}}$ & $69.7^{\mathrm{a}}$ & 2.3 & 0.04 \\
\hline NSP & $10.1^{\mathrm{b}}$ & $14.9^{\mathrm{a}}$ & $16.4^{\mathrm{a}}$ & $21.4^{a}$ & 1.4 & 0.01 \\
\hline \multicolumn{7}{|l|}{ TTD,\% } \\
\hline DM & $75.6^{b}$ & 78.1 & $77.2^{\mathrm{a}}$ & $80.0^{\mathrm{a}}$ & 0.5 & 0.01 \\
\hline Starch & $94.4^{b}$ & $98.6^{a}$ & $97.6^{a}$ & $98.6^{\mathrm{a}}$ & 0.7 & 0.01 \\
\hline GE & $77.8^{\mathrm{b}}$ & $79.8^{\mathrm{a}}$ & $79.8^{\mathrm{a}}$ & $81.1^{\mathrm{a}}$ & 0.7 & 0.01 \\
\hline $\mathrm{CP}$ & $67.1^{\mathrm{b}}$ & $71.2^{\mathrm{a}}$ & $71.6^{\mathrm{a}}$ & $74.2^{\mathrm{a}}$ & 1.0 & 0.01 \\
\hline Phytate & $69.4^{b}$ & $96.8^{\mathrm{a}}$ & $96.3^{\mathrm{a}}$ & $96.0^{\mathrm{a}}$ & 3.2 & 0.01 \\
\hline NSP & $48.9^{b}$ & $61.2^{\mathrm{a}}$ & $59.6^{\mathrm{a}}$ & $66.8^{\mathrm{a}}$ & 1.2 & 0.01 \\
\hline
\end{tabular}

${ }^{1}$ Average initial weight, $7.0 \mathrm{~kg}, 28 \mathrm{~d}$ trial, 6 pigs/trt, ADFI on a DM basis [58]. ${ }^{2}$ Enzyme preparations provided 250 units xylanase, 150 units glucanase, $0.001 \%$ amalyase, $0.0003 \%$ protease, $0.002 \%$ invertase, and 400 units phytase per kilogram of diet and differed in the type of plant cell wall degrading activities. Enzyme A contained cellulase, galactanase, and mannanase; Enzyme $B$ contained cellulase and pectinase; and Enzyme $C$ contained cellulase, galactanase, mannanase, and pectinase.

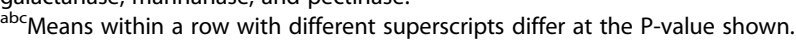


this carbohydrase mixture improved the digestibility of carbohydrates in soybean meal. In a similar manner, supplementation of several multi-enzyme preparations added to corn and soybean meal-based diets (small amounts of wheat, wheat screenings, barley, mill-run, canola meal, and peas) fed to $7 \mathrm{~kg}$ pigs for $28 \mathrm{~d}$, improved growth performance and various nutrient digestibility indices in both the ileum and total tract (Table 1) [58].

Recently, Ji et al. [64] evaluated adding a $\beta$-glucanaseprotease enzyme blend to a corn-soybean meal diet fed to $38 \mathrm{~kg}$ pigs (Table 2). Pigs fed the enzyme blend diet had increased total tract digestibility of DM, energy, CP, TDF, and phosphorus (P), but only increased ileal digestibility of NDF, while ileal digestibility of CP appeared to decrease. These authors suggested that the increase in ileal NDF digestibility (and hemicellulose), with no change in fecal digestibility due to enzyme supplementation, may have shifted some of the digestion of these nutrients from the hindgut to the small intestine, which would avoid the fermentative loss of energy and presumably increase the energetic efficiency of fiber digestion.

Table 2 Effect of enzyme supplementation on percent apparent ileal digestibility (AID) and total-tract digestibility (TTD) of nutrients in 38 kg pigs ${ }^{1}$

\begin{tabular}{|c|c|c|c|c|c|}
\hline \multirow[b]{2}{*}{ AID,\% } & \multicolumn{3}{|c|}{ Diet $^{2}$} & \multicolumn{2}{|c|}{ Statistics } \\
\hline & Basal & $\mathrm{B}+0.05 \%$ & $B+0.10 \%$ & B vs Enz & 0.05 vs 0.10 \\
\hline DM & 70.86 & 69.13 & 70.50 & 0.33 & 0.25 \\
\hline Energy & 70.93 & 69.48 & 70.71 & 0.44 & 0.31 \\
\hline$C P$ & 78.29 & 75.51 & 76.54 & 0.04 & 0.37 \\
\hline Starch & 97.95 & 98.01 & 98.12 & 0.51 & 0.59 \\
\hline NDF & 1.21 & 9.52 & 10.05 & 0.02 & 0.88 \\
\hline ADF & 4.33 & 4.36 & 5.22 & 0.91 & 0.84 \\
\hline TDF & NA & NA & NA & NA & NA \\
\hline Crude fat & 61.40 & 62.94 & 62.18 & 0.49 & 0.68 \\
\hline$P$ & 49.62 & 49.54 & 49.00 & 0.86 & 0.80 \\
\hline \multicolumn{6}{|l|}{ TTD,\% } \\
\hline DM & 87.42 & 88.61 & 88.50 & 0.01 & 0.62 \\
\hline Energy & 86.51 & 87.42 & 87.26 & 0.01 & 0.51 \\
\hline $\mathrm{CP}$ & 86.47 & 88.08 & 87.39 & 0.01 & 0.10 \\
\hline Starch & 99.24 & 99.26 & 99.31 & 0.53 & 0.44 \\
\hline NDF & 54.62 & 55.62 & 56.05 & 0.36 & 0.77 \\
\hline ADF & 64.84 & 61.40 & 65.92 & 0.40 & 0.01 \\
\hline TDF & 60.61 & 65.36 & 65.61 & 0.01 & 0.86 \\
\hline Crude fat & 80.14 & 80.51 & 78.24 & 0.51 & 0.09 \\
\hline$P$ & 53.80 & 61.73 & 57.83 & 0.01 & 0.01 \\
\hline
\end{tabular}

${ }^{1}$ Average initial weight, $38.2 \mathrm{~kg}, 4 \times 4$ Latin Square with $14 \mathrm{~d}$ periods (4 d adapt, $5 \mathrm{~d}$ fecal collection, $3 \mathrm{~d}$ transition, $2 \mathrm{~d}$ ileal collection) [64].

${ }^{2}$ Enzyme contained $660 \beta$-glucanase units/g and 22 hemoglobin units/g.

\section{Enzymes in swine diets containing DDGS}

Spencer et al. [65] reported that adding an enzyme preparation to diets containing 30\% DDGS increased growth performance in nursery pigs. However, the potential benefits of adding enzymes to diets containing

Table 3 Apparent total tract digestibility (\%) of starter pigs fed exogenous feed additives ${ }^{1}$

\begin{tabular}{|c|c|c|c|c|c|c|c|c|}
\hline eat & GE & $\mathrm{N}$ & C & $S$ & $\mathbf{P}$ & ADF & NDF & $\mathrm{EE}$ \\
\hline intr & .2 & 9 & 9 & 78.5 & 60.1 & 1 & 6.6 & 642 \\
\hline 年 & .5 & 77.6 & .4 & . & . & . & 3 & 61.5 \\
\hline alu & 0.01 & 0.01 & 0.01 & 0.17 & 0.01 & 0.01 & 0.01 & 0.14 \\
\hline ictoCel & .0 & 80.4 & 80.3 & 80.1 & 59.8 & 39.4 & 39.3 & 44 \\
\hline value ${ }^{3}$ & 0.14 & 0.55 & 0.42 & 0.03 & 0.79 & 0.76 & 0.15 & 0.66 \\
\hline oPlus2B & 79.5 & 80.3 & 80.0 & 79.6 & 58.7 & 37.7 & 35.0 & 65.0 \\
\hline 3 & 0.59 & 0.64 & 0.85 & 0.17 & 0.24 & 0.31 & 0.39 & 0.64 \\
\hline ona & 3.3 & 78.7 & 79.1 & 77.0 & 54.0 & 35.6 & 25 & 62.8 \\
\hline . & 7 & 0.07 & 0.10 & 4 & 1 & 0.06 & 0.03 & 45 \\
\hline emicel & 78.9 & 79.0 & 79.6 & 79.0 & 59.5 & 36.3 & 33.4 & 65.5 \\
\hline value & 0.53 & 0.17 & 0.48 & 0.49 & 0.60 & 0.12 & 0.09 & 0.45 \\
\hline rzyme & 79.0 & 79.4 & 79.7 & 78.8 & 58.4 & 36 & 35 & 64 \\
\hline value & 0.67 & 0.47 & 0.61 & 0.66 & 0.16 & 0.13 & 0.07 & 0.67 \\
\hline eleezy & 76.9 & 77.4 & 77.7 & 77.3 & 56.1 & 30 & 29. & 61.1 \\
\hline varue & 0.01 & 0.01 & 0.01 & 0.09 & 0.01 & 0.01 & 0.01 & 0.08 \\
\hline ovabio & 80.0 & 80.7 & 80.7 & r & 50 & 38.1 & 65 & 64.4 \\
\hline value & 0.12 & 0.25 & 0.14 & 0.06 & 0.61 & 0.39 & 0.97 & 0.88 \\
\hline Dxazyme & 79.6 & 81.1 & 80.3 & 79.9 & 59.1 & 38. & 39.1 & 63 \\
\hline value & 0.40 & 0.10 & 0.42 & 0.06 & 0.38 & 0.58 & 0.10 & 0.01 \\
\hline PC yeast & 79.6 & 80.1 & 80.3 & 79.4 & 57.9 & 39.0 & 36.4 & 65.9 \\
\hline valu & 40 & 81 & 0.46 & 0.20 & 0.06 & 0.63 & 0.95 & 0.33 \\
\hline \multicolumn{9}{|l|}{ MODEL } \\
\hline value $^{4}$ & .01 & .01 & 0.01 & 0.01 & 0.01 & 0.01 & .01 & 0.08 \\
\hline$=4$ & 0.35 & 0.48 & 0.34 & 0.52 & 0.80 & 1.714 & 1.318 & 1.221 \\
\hline $\mathrm{Nk}-1^{5}$ & 76.9 & 76.0 & 77.6 & 75.4 & 55.3 & 31.4 & 28.5 & 70.6 \\
\hline$k-3$ & 19.2 & 80.1 & 79.8 & 79.3 & 58.9 & 36.2 & 35.8 & 01.9 \\
\hline Wk-5 & 80.5 & 82.4 & 81.2 & 81.8 & 60.0 & 42.0 & 39.1 & 59.4 \\
\hline P value & 0.01 & 0.01 & 0.01 & 0.01 & 0.01 & 0.01 & 0.01 & 0.0 \\
\hline$E^{6}$ & 0.18 & 0.25 & 0.18 & 0.27 & 0.42 & 0.93 & 0.69 & 0.64 \\
\hline
\end{tabular}

${ }^{1}$ Apparent total tract digestibility calculated using indirect marker methodology. There were 16 to 18 individually fed pigs per dietary treatment [66].

${ }^{2}$ Allzyme SSF, 500 mg/kg (Alltech, Lexington, KY); BactoCel, $110 \mathrm{mg} / \mathrm{kg}$ (Lallemand Animal Nutrition, Milwaukee, WI); BioPlus 2B, 500 mg/kg (Chr. Hansen, Milwaukee, WI); Econase XT25, 150 mg/kg (AB Enzymes, Darmstadt, Germany); Hemicel, 500 mg/kg (ChemGen Corp., Gaithersburg, MD); Porzyme 9302, 250 mg/ $\mathrm{kg}$ (Danisco Animal Nutrition, Marlborough, UK); Reelezyme $4 \mathrm{M}, 500 \mathrm{mg} / \mathrm{kg}$ (Prince Agri Products Inc., Quincy, IL); Rovabio AP10, 500 mg/kg (Adisseo, Antony, France); Roxazyme G2G, 220 mg/kg (DSM Nutritional Products Inc., Parsippany,

$\mathrm{NJ}$ ); XPC Yeast, 2,000 mg/kg (Diamond V Mills Inc., Cedar Rapids, IA).

${ }^{3}$ ' $\mathrm{P}$ value' represents comparison of the feed additive to the control diet only.

${ }^{4}$ Model $\mathrm{P}$ and SE value for overall diet effect.

${ }^{5}$ Initial, wk-1, wk-3, and wk-5 BW of $11.88,13.96,23.23$, and 33.26 kg, respectively.

${ }^{6}$ Model $\mathrm{P}$ and SE value for week. 
Table 4 Apparent total tract digestibility (\%) of finisher pigs fed exogenous feed additives ${ }^{1}$

\begin{tabular}{|c|c|c|c|c|c|c|c|c|}
\hline reat & GE & $N$ & C & $S$ & $P$ & ADF & NDF & EE \\
\hline ontrol & 1.4 & 83.8 & 82.3 & 82.7 & 39.0 & 52.9 & 42.1 & 46.5 \\
\hline Izyme & 1 & 84.2 & 83.00 & 83.3 & 46.7 & 56.6 & 169 & 48 \\
\hline 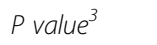 & 27 & 0.61 & 0.29 & 0.38 & 0.01 & 0.08 & 0.08 & 01 \\
\hline ctoCel & 80.8 & 82.3 & 82.0 & 82.4 & 37.1 & 50.1 & 39.5 & 19. \\
\hline alle & 40 & 0.05 & 0.57 & 3 & 0.36 & 19 & 4 & 01 \\
\hline oPlus2B & 81.7 & 83.2 & 82.7 & 82.6 & 39.1 & 56.3 & 45.4 & 38. \\
\hline value ${ }^{3}$ & 0.58 & 0.46 & 0.49 & 0.91 & 0.96 & 0.10 & 0.23 & 0.0 \\
\hline oflase & 80.8 & 82.7 & 81.8 & 83.1 & 39.6 & 50.8 & 42 & 46.7 \\
\hline value & 0.40 & 0.15 & 0.45 & 0.55 & 0.75 & 0.33 & 0.95 & $0.8 z$ \\
\hline - & Oo. & 82. & 81 & 82.4 & 37 & $4 \varepsilon$ & 37 & 44.7 \\
\hline value & 0.30 & 0.20 & 0.27 & 0.74 & 0.37 & 0.03 & 0.08 & 0.2 \\
\hline 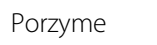 & 9.4 & 80.9 & 00.4 & 80.1 & 33. & 43.8 & 34.0 & $44.2^{2}$ \\
\hline value $^{3}$ & 0.01 & 0.01 & 0.01 & 0.01 & 0.01 & 0.01 & 0.01 & 0.2 \\
\hline ele & 79.5 & 80.7 & 80.4 & 9 & 33.0 & 50 & $3 t$ & 381 \\
\hline value & 0.01 & 0.01 & 0.01 & 0.01 & 0.01 & 0.18 & 0.02 & 0.0 \\
\hline vabio & 81.3 & 83.7 & 82.3 & 82.8 & 36.4 & 52.7 & 43.5 & 45 \\
\hline value ${ }^{3}$ & 0.98 & 0.92 & 0.9 & 0.88 & 0.20 & 0. & 0. & 0.02 \\
\hline oxazyme & 80.9 & 81.9 & 81.7 & 81.9 & 37.4 & 49.8 & 38.1 & 49.9 \\
\hline value & 0.45 & 0.12 & 0 & 0.27 & 0.45 & 0.17 & 0.17 & 0.00 \\
\hline IPC yeast & 80.1 & 82.5 & 81.1 & 82.1 & 35.6 & 50.1 & 38.4 & 43.1 \\
\hline value & 0.05 & 0.10 & 0.5 & 0.36 & 0.09 & 0 & 0 & 0.08 \\
\hline \multicolumn{9}{|l|}{ MODEL } \\
\hline value $e^{4}$ & 0.01 & 0.01 & 0.01 & 0.01 & 0.01 & 0.01 & 0.01 & .01 \\
\hline$=4$ & 0.45 & 0.55 & 0.45 & 0.47 & 1.45 & 1.50 & 1.95 & 1 \\
\hline$k-1^{5}$ & 80.6 & 82.3 & 81.5 & 81.7 & 38.6 & 50.7 & 40.1 & 5.3 \\
\hline 2 & 80.8 & 82.5 & 81.8 & 82.3 & 37.4 & 51.7 & 40.5 & 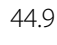 \\
\hline Wk-5 & 81.0 & 83.0 & 82.0 & 82.3 & 36.9 & 50.8 & 40.2 & 44.8 \\
\hline$P$ value ${ }^{6}$ & 0.43 & 0.17 & 0.39 & 0.17 & 0.78 & 0.62 & 0.96 & 89 \\
\hline $\mathrm{SE}^{6}$ & 0.24 & 0.30 & 0.24 & 0.25 & 0.26 & 0.80 & 1.04 & 0.73 \\
\hline
\end{tabular}

${ }^{1}$ Apparent total tract digestibility calculated using indirect marker
methodology. There were 8 individually fed pigs per dietary treatment [66].

methodology. There were 8 individually fed pigs per dietary treatment
${ }^{2}$ Allzyme SSF, $500 \mathrm{mg} / \mathrm{kg}$ (Alltech, Lexington, KY); BactoCel, $110 \mathrm{mg} / \mathrm{kg}$

(Lallemand Animal Nutrition, Milwaukee, WI); BioPlus 2B, $500 \mathrm{mg} / \mathrm{kg}$ (Chr. Hansen,

Milwaukee, WI); Econase XT25, 150 mg/kg (AB Enzymes, Darmstadt, Germany);

Hemicel, 500 mg/kg (ChemGen Corp., Gaithersburg, MD); Porzyme 9302, 250 mg/

$\mathrm{kg}$ (Danisco Animal Nutrition, Marlborough, UK); Reelezyme 4 M, 500 mg/kg

(Prince Agri Products Inc., Quincy, IL); Rovabio AP10, 500 mg/kg (Adisseo, Antony,

France); Roxazyme G2G, 220 mg/kg (DSM Nutritional Products Inc., Parsippany,

NJ); XPC Yeast, 1,000 mg/kg (Diamond V Mills Inc., Cedar Rapids, IA).

3 'P value' represents comparison of the feed additive to the control diet only.

${ }^{4}$ Model $P$ and SE value for overall diet effect.

${ }^{5}$ Initial, wk-1, wk-3, and wk-5 BW of 98.40, 104.90, 119.52, and

$132.20 \mathrm{~kg}$, respectively.

${ }^{6}$ Model P and SE value for week.

increasing levels of corn co-products on growth performance of finishing pigs remains largely unknown.

Recently, we completed an extensive apparent total tract digestibility and performance trial with enzyme/ feed additives commercially available in the United
States [66]. In general, the enzymes contained glucanase, hemicellulase, and/or xylanase activities, the probiotics contained either Pediococcus or Bacillus activities, while the yeast product was derived from Saccharomyces. In general, the products were selected based on their potential to affect energy and fiber digestion, or their ability to modulate the bacterial ecology within the gastrointestinal tract. Basal diets were formulated to be adequate in all nutrients relative to the NRC [67] recommendation for each specific pig weight category over the 5 wk period, and included 30\% DDGS during each phase of growth. TIME EFFECT: In the starter experiment, digestibility of GE, N, carbon (C), sulfur (S), ADF, NDF and ether extract (EE) increased from week-1 to week- 5 suggesting that the gastrointestinal tract of the $12 \mathrm{~kg}$ pig adapts to dietary fiber from DDGS and nutrient digestibility improves with continuous feeding over time. This finding is consistent with the increased ability of the digestive system in growing pigs to digest nutrients (especially fiber) with increasing age. In contrast, nutrient digestibility did not improve from week-1 to week-5 in finishing pigs. FEED ADDITIVE EFFECT: While the results of this research indicate that some of the feed additive products evaluated had variable, but small effects on nutrient digestibility (Tables 3 and 4),

Table 5 Performance of pigs fed exogenous feed additives ${ }^{1}$

\begin{tabular}{|c|c|c|c|c|c|c|}
\hline \multirow[b]{2}{*}{ Treatment $^{2}$} & \multicolumn{3}{|c|}{ Starter, 12 to $33 \mathrm{~kg} \mathrm{BW}$} & \multicolumn{3}{|c|}{ Finisher, 98 to $132 \mathrm{~kg} \mathrm{BW}$} \\
\hline & ADG, $\mathbf{k g}$ & ADFI, kg & $\mathrm{G}: \mathrm{F}$ & ADG, kg & ADFI, kg & $\mathrm{G}: \mathrm{F}$ \\
\hline Control & 0.640 & 1.126 & 0.572 & 0.999 & 3.032 & 0.333 \\
\hline Allzyme & 0.651 & 1.140 & 0.574 & 0.961 & 3.118 & 0.311 \\
\hline actoCel & 0.615 & 1.083 & 0.568 & 1.007 & 3.084 & 0.328 \\
\hline BioPlus2B & 0.645 & 1.162 & 0.559 & 0.988 & 3.179 & 0.315 \\
\hline Econase & 0.653 & 1.133 & 0.578 & 1.051 & 3.240 & 0.325 \\
\hline Hemicel & 0.629 & 1.149 & 0.551 & 0.933 & 3.239 & 0.292 \\
\hline Porzyme & 0.642 & 1.131 & 0.570 & 0.979 & 3.077 & 0.318 \\
\hline Releezyme & 0.639 & 1.109 & 0.579 & 0.983 & 3.115 & 0.311 \\
\hline Rovabio & 0.648 & 1.148 & 0.565 & 0.906 & 2.985 & 0.302 \\
\hline Roxazyme & 0.638 & 1.100 & 0.583 & 0.975 & 3.084 & 0.321 \\
\hline $\mathrm{XPC}$ yeast & 0.653 & 1.157 & 0.568 & 0.862 & 2.930 & 0.294 \\
\hline \multicolumn{7}{|l|}{ MODEL } \\
\hline$P$ value & 0.87 & 0.70 & 0.72 & 0.60 & 0.90 & 0.56 \\
\hline SE & 0.016 & 0.030 & 0.011 & 0.057 & 0.141 & 0.014 \\
\hline
\end{tabular}

${ }^{1}$ Performance over the 5 -wk period. There were $16-18$ and 8 individually fed pigs per treatment in the starter and finisher phase, respectively [66].

${ }^{2}$ Allzyme SSF, 500 mg/kg (Alltech, Lexington, KY); BactoCel, 110 mg/kg (Lallemand Animal Nutrition, Milwaukee, WI); BioPlus $2 B, 500 \mathrm{mg} / \mathrm{kg}$ (Chr. Hansen, Milwaukee, WI); Econase XT25, 150 mg/kg (AB Enzymes, Darmstadt, Germany); Hemicel, 500 mg/kg (ChemGen Corp., Gaithersburg, MD); Porzyme 9302, $250 \mathrm{mg} / \mathrm{kg}$ (Danisco Animal Nutrition, Marlborough, UK);

Reelezyme 4 M, $500 \mathrm{mg} / \mathrm{kg}$ (Prince Agri Products Inc., Quincy, IL); Rovabio AP10, $500 \mathrm{mg} / \mathrm{kg}$ (Adisseo, Antony, France); Roxazyme G2G, $220 \mathrm{mg} / \mathrm{kg}$ (DSM Nutritional Products Inc., Parsippany, NJ); XPC Yeast, 2,000 mg/kg starter or $1,000 \mathrm{mg} / \mathrm{kg}$ finisher (Diamond V Mills Inc., Cedar Rapids, IA). 
Table 6 Growth performance and apparent total tract digestibility of 10 to $23 \mathbf{k g}$ pigs receiving phytase, or a cocktail of xylanase, amylase, and protease ${ }^{1}$

\begin{tabular}{|c|c|c|c|c|c|c|c|}
\hline \multirow[b]{2}{*}{ Dietary treatment } & \multicolumn{3}{|c|}{ Pig performance } & \multicolumn{4}{|c|}{ Apparent total tract digestibility,\% } \\
\hline & ADG, $g$ & ADFI, $g$ & G:F, g:kg & DM & GE & $\mathrm{N}$ & $\mathbf{P}$ \\
\hline Negative control (NC) & 398 & 1140 & 363 & 80.2 & 79.8 & 80.1 & 38.3 \\
\hline $\mathrm{NC}+$ Phytase $^{2}$ & 483 & 1070 & 457 & 80.1 & 78.1 & 80.2 & 49.9 \\
\hline $\mathrm{NC}+$ Enzyme $^{3}$ & 393 & 1050 & 380 & 82.3 & 80.1 & 81.2 & 48.3 \\
\hline $\mathrm{NC}+\mathrm{Ph}+\mathrm{En}$ & 479 & 1210 & 415 & 80.0 & 79.0 & 80.0 & 51.1 \\
\hline SEM & 10.4 & 30 & 13.7 & 0.20 & 0.43 & 0.43 & 0.87 \\
\hline
\end{tabular}

${ }^{1}$ There were 4 replicate pens each of barrows and gilts ( 1 pig/pen) in the $28 \mathrm{~d}$ trial.

${ }^{2}$ Phytase was added at the rate of 500 phytase units $/ \mathrm{kg}$ diet [78].

${ }^{3}$ Cocktail of $400 \mathrm{U}$ of xylanase, $4,000 \mathrm{U}$ of amylase, and 2,500 U of protease per $\mathrm{kg}$ of diet.

none of the products were effective in improving starter and finishing pig growth performance (Table 5) when fed nutritionally adequate corn-soy diets containing 30\% DDGS. Many of the enzyme/additive products evaluated in this study contained ingredients that should have been effective in for improving energy/fiber digestibility in 30\% DDGS diets. We did not, however, confirm the specified enzyme/active ingredient activity for these additives as it may be possible that they did not contain enough activity to provide significant improvements in digestibility for many of the nutrients evaluated. In addition, because these diets were formulated to meet the nutrient needs of pigs in each growth phase evaluated, the improvements or decreases in nutrient digestibility that did occur were too small to influence overall pig performance.

Unfortunately, results of studies where there are no effects of supplemental enzymes on pig growth performance often are not published in the scientific literature, leading to a publication bias in the information being available to pork producers, swine nutritionists, and other pork industry professionals.

\section{Phytase alone or in combination with other enzymes}

The impact of dietary phytase supplementation on the digestibility of energy has not been consistent. While most studies [68-72] have observed no impact of phytase on energy digestibility, others [73-76] have reported positive effects. Recent results from Kerr et al. [77] were

Table 7 Concentration of energy in corn and 10 sources of corn distillers dried grains with solubles (DDGS) fed to growing pigs ${ }^{1}$

\begin{tabular}{lccccc}
\hline & & \multicolumn{4}{c}{ DDGS } \\
\cline { 3 - 6 } Item & Corn & Average & SD & $\begin{array}{c}\text { Lowest } \\
\text { value }\end{array}$ & $\begin{array}{c}\text { Highest } \\
\text { value }\end{array}$ \\
\hline $\mathrm{GE}_{1} \mathrm{kcal} / \mathrm{kg} \mathrm{DM}$ & 4,496 & 5,434 & 108 & 5,272 & 5,592 \\
$\mathrm{ATTD}^{2}$ of energy,\% & 90.4 & 76.8 & 2.73 & 73.9 & 82.8 \\
$\mathrm{DE}, \mathrm{kcal} / \mathrm{kg} \mathrm{DM}$ & 4,088 & 4,140 & 205 & 3,947 & 4,593 \\
$\mathrm{ME}, \mathrm{kcal} / \mathrm{kg} \mathrm{DM}$ & 3,989 & 3,897 & 210 & 3,674 & 4,336 \\
\hline
\end{tabular}

${ }^{1}$ Data from Pedersen et al. [83] as adapted from Stein and Shurson [82].

${ }^{2}$ ATTD $=$ apparent total tract digestibility. also inconclusive, suggesting that if there is an effect of phytase on energy digestibility, it is relatively small in magnitude and highly variable.

Data relative to the impact of phytase, with or without other enzymes, on nutrient (and energy) digestibility is lacking. Olukosi et al. [78] supplemented diets comprised of corn, wheat midds, soybean meal, and canola meal with either phytase or an enzyme cocktail (xylanase, amylase, and protease) alone, or in combination, and fed them to 10 to $23 \mathrm{~kg}$ pigs (Table 6). These data suggest that even though phytase improved pig gain and feed efficiency, addition of the enzyme cocktail, alone or in combination with phytase, had no impact on pig performance. Neither the addition of phytase nor the enzyme cocktail, alone or in combination, had any consistent effect on DM, energy, or $\mathrm{N}$ digestibility, but each improved $\mathrm{P}$ digestibility. The effects, however, were not additive. In an additional experiment using wheat to replace corn in the diet ( 23 to $52 \mathrm{~kg} \mathrm{BW}, 42 \mathrm{~d}$ trial), there were no effects of phytase or xylanase (500 $\mathrm{U}$ and 4,000 $\mathrm{U} / \mathrm{kg}$, respectively) on pig performance, or on $\mathrm{N}$ and energy digestibility [78]. Phytase, but not xylanase, improved phosphorus digestibility as one would expect from an enzyme that releases phosphate.

Table 8 Concentration of carbohydrates and apparent total tract digestibility (ATTD) of dietary fiber in corn distillers dried grains with solubles ${ }^{1}$

\begin{tabular}{lcccc}
\hline Item & Average & Low value & High value & SD \\
\hline Starch, total,\% & 7.3 & 3.8 & 11.4 & 1.4 \\
Starch, soluble,\% & 2.6 & 0.5 & 5.0 & 1.2 \\
Starch, insoluble,\% & 4.7 & 2.0 & 7.6 & 1.5 \\
ADF,\% & 9.9 & 7.2 & 17.3 & 1.2 \\
NDF,\% & 25.3 & 20.1 & 32.9 & 4.8 \\
Insoluble TDF,\% & 35.3 & 26.4 & 38.8 & 4.0 \\
Soluble TDF,\% & 6.0 & 2.36 & 8.54 & 2.1 \\
TDF,\% & 42.1 & 31.2 & 46.3 & 4.9 \\
ATTD of TDF,\% & 43.7 & 23.4 & 55.0 & 10.2 \\
\hline
\end{tabular}

${ }^{1} \mathrm{~N}=46$ for data on starch, ADF, and NDF; $n=8$ for data on insoluble, soluble, and total dietary fiber [82]. 
Table 9 Analyzed composition of corn co-products, DM basis ${ }^{1}$

\begin{tabular}{|c|c|c|c|c|c|c|c|c|c|}
\hline Item & DDGS (WI) & DDGS (IA) & DDGS (SD) & RO-DDGS (SD) & DDGS (BPX) & Drum-DDGS (MN) & $\begin{array}{l}\text { Microwave-DDGS } \\
\text { (MN) }\end{array}$ & $\begin{array}{c}\text { Dried } \\
\text { solubles }\end{array}$ & $\begin{array}{l}\text { Gluten } \\
\text { feed }\end{array}$ \\
\hline Crude protein & 29.62 & 29.65 & 31.94 & 34.74 & 29.49 & 32.69 & 34.12 & 23.75 & 24.29 \\
\hline Starch & 7.85 & 3.47 & 6.24 & 3.04 & 4.94 & 2.12 & 1.05 & 6.34 & 12.57 \\
\hline Crude fiber & 7.05 & 7.76 & 7.56 & 8.69 & 7.95 & 7.93 & 8.35 & 0.08 & 8.56 \\
\hline TDF & 30.34 & 38.14 & 35.69 & 37.20 & 35.90 & 35.38 & 43.18 & 16.07 & 40.07 \\
\hline NDF & 34.61 & 40.13 & 40.12 & 50.96 & 33.41 & 44.87 & 49.12 & 2.33 & 42.66 \\
\hline ADF & 11.25 & 10.55 & 14.42 & 15.82 & 8.62 & 13.16 & 14.66 & 0.49 & 9.90 \\
\hline Cellulose & 10.64 & 10.12 & 11.72 & 12.72 & 8.21 & 11.95 & 13.37 & 0.79 & 9.17 \\
\hline Lignin & 1.21 & 1.06 & 3.16 & 3.49 & 1.00 & 1.72 & 1.92 & 0.31 & 1.05 \\
\hline Item & $\begin{array}{l}\text { DHDG } \\
\text { corn }\end{array}$ & $\begin{array}{l}\text { Dehy corn } \\
\text { germ }\end{array}$ & $\begin{array}{c}\text { Corn germ } \\
\text { meal }\end{array}$ & Bran & Bran + solubles & Gluten meal & HP-DDG (MOR) & $\begin{array}{l}\text { HP-DDG } \\
\text { (Poet) }\end{array}$ & $\begin{array}{l}\text { HP-DDG } \\
\text { (ICM) }\end{array}$ \\
\hline Starch & 87.96 & 25.00 & 15.29 & 23.25 & 25.73 & 11.08 & 0.51 & 7.30 & 5.10 \\
\hline Crude fiber & 0.60 & 4.87 & 10.69 & 11.54 & 4.80 & 1.44 & 8.14 & 9.42 & 7.87 \\
\hline TDF & 2.61 & 24.78 & 47.76 & 53.60 & 26.65 & 9.24 & 28.80 & 31.28 & 36.75 \\
\hline NDF & 4.27 & 27.37 & 61.05 & 56.86 & 25.21 & 12.25 & 43.52 & 32.00 & 51.09 \\
\hline ADF & 0.49 & 6.13 & 12.49 & 13.14 & 5.35 & 7.57 & 25.42 & 12.61 & 15.11 \\
\hline Cellulose & 0.77 & 5.21 & 11.71 & 12.78 & 5.38 & 5.95 & 22.55 & 12.05 & 14.25 \\
\hline Lignin & 0.33 & 1.28 & 1.22 & 0.89 & 0.55 & 2.24 & 3.40 & 0.95 & 1.44 \\
\hline
\end{tabular}

${ }^{1}$ Abbreviations: TDF, total dietary fiber; NDF, neutral detergent fiber; ADF, acid detergent fiber; RO-DDGS, reduced oil-DDGS; drum- or microwave-dried DDGS; DHDG, dehulled-degermed; HP-DDG, high protein dried distillers grains. Abbreviations within brackets ( ) refers to the state or company where the product was obtained [84].

Results from experiments evaluating the impact of phytase, with or without other enzymes, on nutrient (and energy) digestibility in diets containing DDGS are also lacking and inconsistent. While addition of 500 units phytase improved P digestibility in diets containing 20\% DDGS in starter or finisher pigs, it did not improve DM digestibility $[79,80]$. In contrast, Lindemann et al. [81] reported that 64 to $123 \mathrm{~kg}$ pigs fed diets containing $20 \%$ DDGS supplemented with 250 or $500 \mathrm{U} / \mathrm{kg}$ phytase exhibited greater DM, energy, and $\mathrm{N}$ digestibility than unsupplemented pigs, but there were no further improvements in fecal DM, energy or N digestibility when xylanase was added in addition to the phytase addition.

\section{Energy and Fiber in Corn Co-products}

Gross energy in DDGS averages 5,434 kcal/kg DM and is greater than the concentration of GE in corn (Table 7) [82]. However, the digestibility of energy, measured as a percentage of GE, is lower in DDGS than in corn [82]. The digestible energy (DE) and metabolizable energy (ME) content of DDGS is 4,140 and 3,897 $\mathrm{kcal} / \mathrm{kg} \mathrm{DM}$, respectively [83], which are similar to the $\mathrm{DE}$ and $\mathrm{ME}$ content in corn (Table 7). The net energy value of DDGS has not been determined, but research is currently underway to measure these values.

Since most of the starch in corn has been converted to ethanol, DDGS contains approximately 35\% insoluble and $6 \%$ soluble dietary fiber [82] (Table 8). Likewise, most corn co-products have a high amount of insoluble fiber which can be observed by comparing the relatively similar TDF and NDF concentrations in these co-products [84] (Table 9). Furthermore, corn "fiber" has a large hemicellulose component as defined by the difference between NDF and ADF. These results are similar to those reported by Leathers [85], where the corn fiber composition from six studies representing different geographic regions showed that hemicellulose is the predominant constituent in corn fiber, followed by xylose (Table 10).

The apparent total tract digestibility of dietary fiber in DDGS averages $43.7 \%$, but ranges from $23 \%$ to $55 \%$. This variation in fiber digestibility is believed to influence digestibility of energy in DDGS. Apparent ileal digestibility and total tract digestibility of dietary fiber in DDGS is

Table 10 Major components of corn fiber

\begin{tabular}{lcccccc}
\hline & \multicolumn{5}{c}{ Geographic location } \\
\cline { 2 - 7 } Component & A & B & C & D & E & F \\
\hline Starch & 22 & 11 & 18 & 22 & 20 & 23 \\
Hemicellulose & 40 & 53 & 32 & 47 & 29 & 39 \\
Xylose & 24 & 25 & 20 & 28 & 18 & 19 \\
Arabinose & 16 & 18 & 10 & 19 & 11 & 11 \\
Cellulose & 12 & 18 & 24 & ND & 14 & ND \\
Protein & 12 & 11 & ND & ND & 11 & 12 \\
\hline
\end{tabular}

$\mathrm{ND}=$ not determined $[85]$. 
Table 11 Effect of $\beta$-glucanase supplementation on energy digestibility

\begin{tabular}{lcccccccc}
\hline & \multicolumn{3}{c}{ Diet composition,\% } & & \multicolumn{3}{c}{$\boldsymbol{\beta}$-glucanase supplementation,\% } \\
\cline { 2 - 3 } Diet & NDF & ADF & $\boldsymbol{\beta}$-glucans & & $\mathbf{0}$ & $\mathbf{0 . 0 5}$ & $\mathbf{0 . 1 0}$ & $\mathbf{0 . 2 0}$ \\
\hline Barley-SBM & 8.4 & 2.3 & 3.2 & & 85.2 & 87.8 & 86.4 & 88.5 \\
Wheat-SBM & 7.9 & 2.5 & 0.8 & & 86.8 & 88.1 & 88.4 & 88.4 \\
Corn-SBM & 8.1 & 1.9 & 0.3 & & 85.8 & 84.4 & 83.8 & 85.7 \\
Rye-SBM & 7.4 & 2.1 & 0.7 & & 87.2 & 88.0 & 88.1 & 87.1 \\
\hline
\end{tabular}

[61].

higher than in corn, and are presumed to be improved as a result of the processing and fermentation processes used in ethanol plants [86]. However, less than $50 \%$ of total dietary fiber is fermented over the entire digestive tract, indicating that more than $50 \%$ passes through pigs without being fermented [86]. As a result, there is a significant amount of non-fermented carbohydrate in DDGS that could potentially be utilized to a greater extent if appropriate exogenous enzymes can be developed to enhance the utilization of these substrates in DDGS diets.

Consequently, when evaluating the effectiveness of exogenous enzymes, the composition of "fiber" must be considered in order for energy and nutrient digestibility to potentially be improved. This is clearly demonstrated by Li et al., [61] who evaluated the effectiveness of adding $\beta$-glucanase to a broad range of diets, differing largely in $\beta$-glucan content. Their data showed that supplementation of $\beta$-glucanase had no effect on energy digestibility in wheat-, corn-, or rye-soybean meal diets, but did improve energy digestibility in barley-soybean meal diets (Table 11), which reflected the dietary differences in $\beta$-glucan concentrations.

\section{Enzyme activity and substrates}

It is clear that there needs to be an improved characterization of fibrous components in all feedstuffs [2]. Likewise, there needs to be some agreement on key enzyme activities and analysis of these activities so that a scientific evaluation of enzyme/feed additive products

Table 12 Key enzyme activity and associative substrate

\begin{tabular}{cc}
\hline Activity & Substrate \\
\hline Xylanase & Arabino-xylose (NSP) \\
$\beta$-Glucanase & $\beta$-Glucans (NSP) \\
Mannase & Oligo-mannans (Mannose) \\
a-Galactosidase & a-Galactosyl moieties \\
Cellulase & Cellulose (a-glucans) \\
Pectanase & Pectins (NSP) \\
Amylase & Amylose \\
Protease & Proteins \\
Phytase & Phytic acid \\
\hline
\end{tabular}

can be achieved. Lastly, a better understanding of enzyme-substrate relationships combined with an improved understanding of gastrointestinal physiology in relation to enzyme-substrate will improve our understanding of when exogenous feed enzymes will likely have a significant, positive response, with a listing of key enzymes listed in Table 12.

\section{Conclusions}

Application of enzymes in an effort to improve nutrient digestibility of plant-based feed ingredients for swine and poultry has been studied for decades. However, with a large diversity and concentration of chemical characteristics existing among plant-based feed ingredients, as well as interactions among constituents within feed ingredients and diets, improvements in nutrient digestibility and pig performance from adding exogenous enzymes to growing pig diets depends on understanding these characteristics in relation to enzyme activity. Essentially, the enzyme must match the target substrate(s), there may need to be a 'cocktail' of enzymes to effectively breakdown the complex matrixes of fibrous carbohydrate structures, and there must be some negative role that these substrates have on nutrient digestibility or voluntary feed intake. With the inverse relationship between fiber content and energy digestibility being well described for several feed ingredients, it is only logical that development of enzymes that degrade fiber, and thereby improve energy digestibility or voluntary feed intake will have a greater likelihood to be beneficial to improve fiber utilization in swine, both metabolically and economically. The results of an unpublished study by the authors suggests that although some of the enzyme/additive products evaluated had variable, but small effects on nutrient digestibility, none of these products were effective in improving starter and finishing pig growth performance when fed nutritionally adequate corn-soy diets containing 30\% DDGS.

\footnotetext{
Abbreviations

ADF: Acid detergent fiber; ADG: Average daily gain; ADFI: Average daily feed intake; AID: Apparent ileal digestibility; ATTD: Apparent total tract digestibility; C: Carbon; CP: Crude protein; DE: Digestible energy; DDGS: Dried distillers grains with soluble; DM: Dry matter; EE: Ether extract; GE: Gross energy; GF: Gain to feed ratio; ME: Metabolizable energy; N: Nitrogen; NDF: Neutral detergent fiber; NSP: Nonstarch polysaccharides; P: Phosphorus; S: Sulfur; TDF: Total dietary fiber; TTD: Total-tract digestibility; VFA: Volatile fatty acids.

Competing interests

The authors declared that they have no competing interests.

Authors' contributions

BJK and GCS co-wrote this review and any internal research reported was jointly designed and interpreted. Both authors have read and approved the manuscript.

Authors' information

Brian J. Kerr, Ph.D., is a Lead Scientist/Animal Scientist for the USDA Agricultural Research Service, with expertise in digestibility, nutrient utilization, and
} 
evaluation of alternative feed ingredients. Gerald C. Shurson, Ph.D., is a Professor of Animal Science at the University of Minnesota with expertise in nutrition and the use of alternative feed ingredients in swine production.

Mention of a trade name, proprietary product, or specific equipment does not constitute a guarantee or warranty by the USDA or the University of Minnesota and does not imply approval to the exclusion of other products that may be suitable. The USDA is an equal opportunity provider and employer

\section{Author details}

'USDA-ARS-National Laboratory for Agriculture and the Environment, Ames, IA 50011, USA. ${ }^{2}$ University of Minnesota, St. Paul, MN 55108, USA.

Received: 1 October 2012 Accepted: 17 December 2012

Published: 15 March 2013

\section{References}

1. Slominski BA: Carbohydrates in feedstuffs in relation to dietary enzyme use. In Proc. 12th West. Nutr. Conf. Edited by. Winnipeg, Manitoba, Canada: University of Manitoba Press; 1991:140-162.

2. NRC: Nutrient Requirements of Swine. 10th rev. ed. Washington, D.C: Natl. Acad. Press; 2012.

3. Whitney MH, Shurson GC: Growth performance of nursery pigs fed diets containing increasing levels of corn distiller/s dried grains with solubles originating from a modern Midwestern ethanol plant. J Anim Sci 2004, 82:122-128

4. Weber TE, Ziemer CJ, Kerr BJ: Effects of adding fibrous feedstuffs to the diet of young pigs on growth performance, intestinal cytokines, and circulating acute-phase proteins. J Anim Sci 2008, 86:871-881.

5. Whitney MH, Shurson GC, Johnston L, Wulf DM, Shanks BC: Growth performance and carcass characteristics of grower-finisher pigs fed highquality corn distillers dried grain with solubles originating from a modern Midwestern ethanol plant. J Anim Sci 2006, 84:3356-3363.

6. Muley NS, van Heugten E, Moeser AJ, Rausch KD, van Kempen TATG Nutritional value for swine of extruded corn and corn fractions obtained after dry milling. J Anim Sci 2007, 85:1695-1701.

7. NRC: Nutrient Requirements of Horses. 6th rev. ed. Washington, D.C: Natl. Acad. Press; 2007.

8. Grieshop CM, Reece DE, Fahey GC: Nonstarch polysaccharides and oligosaccharides in swine nutrition. In Swine Nutrition. 2nd edition. Edited by Lewis AJ, Southern LL. Boca Raton, FL: CRC Press; 2001:107-130.

9. Selvendran RR, Robertson JA: The chemistry of dietary fibre: a holistic view of the cell wall matrix. In Dietary Fibre: Chemical and Biological Aspects. Edited by Southgate DAT, Waldron K, Johnson IT, Fenwick GR. Cambridge: Royal Soceity of Chemistry Special Publication No 83. Royal Society of Chemistry; 1990:27-43.

10. Bach Knudsen KE, Hansen I: Gastrointestinal implications in pigs of wheat and oat fractions. 1. Digestibility and bulking properties of polysaccharides and other major constituents. Br J Nutr 1991, 65:217-232.

11. Fadel JG, Newman RK, Newman CW, Graham H: Effects of baking hulless barley on the digestibility of dietary components as measured at the ileum and in the feces in pigs. J Nutr 1989, 119:722-726.

12. Stanogias G, Pearce GR: The digestion of fibre by pigs. 3. Effects of the amount and type of fibre on physical characteristics of segments of the gastrointestinal tract. Br J Nutr 1985, 53:537-548.

13. Goodlad JS, Mathers JC: Digestion by pigs of non-starch polysaccharides in wheat and raw peas (Pisum sativum) fed in mixed diets. Br J Nutr 1991, 65:259-270.

14. Farrell DJ, Johnson KA: Utilization of cellulose by pigs and its effects on caecal function. Anim Prod 1970, 14:209-217.

15. Imoto S, Namioka S: VFA production in the pig large intestine. J Anim Sci 1978, 47:467-478

16. Van Kass ML, Soest PJ, Pond WG, Lewis B, McDowell RE: Utilization of dietary fiber from alfalfa by growing swine. I. Apparent digestibility of diet components in specific segments of the gastrointestinal tract. J Anim Sci 1980, 50:175-191.

17. Latymer EA, Low AG: Tissue incorporation and excretion of ${ }^{14} \mathrm{C}$ in pigs after injection of $\left[1-1^{4} \mathrm{C}\right]$ or $\left[2-1^{4} \mathrm{C}\right]$ propionic acid into the caecum. Proc Nutr Soc 1987, 43:12A.

18. Rérat A, Fiszlewicz M, Giusi A, Vaugelade P: Influence of meal frequency on postprandial variations in the production and absorption of volatile fatty acids in the digestive tract of conscious pigs. J Anim Sci 1987, 64:448-456.

19. Yen JT: Oxygen consumption and energy flux of porcine splanchnic tissues. Digestive Physiology in Pigs, Proc. of the VII International Symposium on Digestive Physiology in Pigs. EAAP Publ 1997, 88:260-269.

20. Giusi-Perier A, Fiszlewicz M, Rérat A: Influence of diet composition on intestinal volatile fatty acid and nutrient absorption in unanesthetized pigs. J Anim Sci 1989, 67:386-402.

21. Noblet J, Fortune H, Shi XS, Dubois S: Prediction of net energy value of feeds for growing pigs. J Anim Sci 1994, 72:344-354.

22. Anugwa FOI, Varel VH, Dickson JS, Pond WG, Krook LP: Effects of dietary fiber and protein concentration on growth, feed efficiency, visceral organ weights and large intestine microbial populations of swine. J Nutr 1989, 119:879-886.

23. Jørgensen $H$, Zhao $X Q$, Eggum BO: The influence of dietary fiber and environmental temperature on the development of the gastrointestinal tract digestibility, degree of fermentation in the hind-gut and energy metabolism in pigs. Br J Nutr 1996, 75:365-378.

24. Jin L, Reynolds LP, Redmer DA, Caton JS, Crenshaw JD: Effects of dietary fiber on intestinal growth, cell proliferation, and morphology in growing pigs. J Anim Sci 1994, 72:2270-2278.

25. Howard MD, Gordon DT, Pace LW, Garleb KA, Kerley MS: Effect of dietary supplementation of fructooligosaccharides on colonic microbiota populations and epithelial cell proliferation in neonatal pigs. J Pediatr Gastroenterol Nutr 1995, 21:297-303.

26. Wenk $C$ : The role of dietary fibre in the digestive physiology of the pig. Anim Feed Sci Tech 2001, 90:21-33.

27. Zebrowska T, Low AG, Zebrowska H: Studies on gastric digestion of protein and carbohydrate, gastric secretion and exocrine pancreatic in the growing pig. Br J Nutr 1983, 49:401-410.

28. Johansen HN, Bach Knudsen KE, Sandström B, Skjøth F: Effects of varying content of soluble dietary fiber from wheat flour and oat milling fractions on gastric emptying in pigs. Br J Nutr 1996, 75:339-351.

29. Rainbird AL: Effect of guar gum on gastric emptying of test meals of varying energy content in growing pigs. Br J Nutr 1986, 55:99-109.

30. Rainbird AL, Low AG: Effect of various types of dietary fiber on gastric emptying in growing pigs. Br J Nutr 1986, 55:111-121.

31. Rijnen MM, Heetkamp JW, Verstegen MW, Schrama JW: Effects of dietary fermentable carbohydrates on physical activity and energy metabolism in group-housed sows. J Anim Sci 1999, 77(Suppl. 1):182. Abstr.

32. Schulze $H$, van Leeuwen $P$, Verstegen MWA, van den Berg JWO: Dietary level and source of neutral detergent fiber and ileal endogenous nitrogen flow in pigs. J Anim Sci 1995, 73:441-448.

33. Potkins ZV, Lawrence TL, Thomlinson JR: Effects of structural and nonstructural polysaccharides in the diet of the growing pig on gastric emptying rate and rate of passage of digesta to the terminal ileum and through the total gastrointestinal tract. Br J Nutr 1991, 65:391-413.

34. Bardon T, Fioramonti J: Nature of the effects of bran on digestive transit time in pigs. Br J Nutr 1983, 50:685-690.

35. Borel $P$, Lairon $D$, Senft $M$, Chautan $M$, Lafont $H$ : Wheat bran and wheat germ: effect on digestion and intestinal absorption of dietary lipids in the rat. Am J Clin Nutr 1989, 49:1192-1202.

36. Kornegay ET, Moore RJ: Dietary fiber sources may affect mineral use in swine. Feedstuffs 1986, 58:36-49.

37. Teitge DA, Campbell GL, Classen HL, Thacker PA: Heat pretreatment as a means of improving the response to dietary pentosanase in chicks fed rye. Can J Anim Sci 1991, 71:507-513.

38. Brenes A, Marquardt RR, Guenter W, Rotter BA: Effect of enzyme supplementation on the nutritional value of raw, autoclaved, and dehulled lupins (Lupinus albus) in chicken diets. Poult Sci 1993, 72:2281-2293.

39. Brenes A, Rotter B: A., Marquardt, R. R., Guenter, W. The nutritional value of raw, autoclaved and dehulled peas (Pisum satvum L.) in chicken diets as affected by enzyme supplementation. Can J Anim Sci 1993, 73:605-614

40. Graham H, Fadel JG, Newman CW, Newman RK: Effect of pelleting and B-glucanase supplementation on the ileal and fecal digestibility of a barley-based diet in the pig. J Anim Sci 1989, 67:1293-1298.

41. Poel van der AFB, Gravendeel S, van Kleef DJ, Jansman AJM, Kemp B: Tannin-containing fava beans (Vicia faba L.): effects of methods of 
processing on ileal digestibility of protein and starch for growing pigs. Anim Feed Sci Tech 1992, 36:205-214.

42. Thacker PA, Campbell GL: Performance of growing/finishing pigs fed untreated or micronized hulless barley-based diets with or without B-glucanase. J Anim Feed Sci 1999, 8:157-170.

43. Nyachoti CM, Arntfield SD, Guenter W, Cenkowski S, Opapeju FO: Effect of micronized pea and enzyme supplementation on nutrient utilization and manure output in growing pigs. J Anim Sci 2006, 84:2150-2156.

44. Yen JT, Baker DH, Harmon BG, Jensen AH: Corn gluten feed in swine diets and effect of pelleting on tryptophan availability to pigs and rats. J Anim Sci 1971, 33:987-991.

45. Hancock JD, Behnke KC: Use of ingredient and diet processing technologies (grinding, mixing, pelleting, and extruding) to produce quality feeds for pigs. In Swine Nutrition. 2nd edition. Edited by Lewis AJ, Southern LL. Boca Raton, FL: CRC Press; 2001:469-498.

46. Stark CR: Feed processing to maximize feed efficiency. In Feed Efficiency in Swine. Edited by Patience JF. Wageningen: Wageningen Academic Publishers; 2012:131-151

47. Chesson A: Supplementary enzymes to improve the utilization of pig and poultry diets. In Recent Advances in Animal Nutrition. Edited by Haresign W, Cole DJA. London: Butterworths; 1987:71-89.

48. Bedford MR: Exogenous enzymes in monogastric nutrition-their current value and future benefits. Anim Feed Sci Tech 2000, 86:1-13.

49. Annison G, Choct M: Anti-nutritive activities of cereal non-starch polysaccharides in broiler diets and strategies minimizing their effects. Worldıs Poul Sci J 1991, 47:232-242.

50. Cowan WD: Understanding the manufacturing, distribution, application, and overall quality of enzymes in poultry feeds. J Appl Poult Res 1993, 2:93-99.

51. Aimonen EMJ, Nasi M: Replacement of barley by oats and enzyme supplementation in diets for laying hens. 1. Performance and balance trial results. Acta Agric Scand 1991, 41:179-192.

52. Thacker PA, Campbell GL, GrootWassink JWD: Effect of salinomycin and enzyme supplementation on nutrient digestibility and the performance of pigs fed barley- or rye-based diets. Can J Anim Sci 1992, 72:117-125.

53. Viveros A, Brenes A, Pizarro M, Castano M: Effect of enzyme supplementation of a diet based on barley, and autoclave treatment, on apparent digestibility, growth performance and gut morphology of broilers. Anim Feed Sci Tech 1994, 48:237-251.

54. Hubener K, Vahjen W, Simon O: Bacterial responses to different dietary cereal types and xylanase supplementation in the intestine of broiler chicken. Arch Anim Nutr 2002, 56:167-187.

55. Saleh F, Tahir M, Ohtsuka A, Hayashi K: A mixture of pure cellulose, hemicellulase and pectinase improved broiler performance. Br Poult Sci 2005, 46:602-606.

56. Inborr J, Schmitz M, Ahrens F: Effect of adding fibre and starch degrading enzymes to a barley/wheat based diet on performance and nutrient digestibility in different segments of the small intestine of early weaned pigs. Anim Feed Sci Tech 1993, 44:113-127.

57. Nonn $\mathrm{H}$, Kluge $\mathrm{H}$, Jeroch $\mathrm{H}$, Broz J: Effects of carbohydrate-hydrolysing enzymes in weaned piglets fed diets based on peas and wheat. Agribiol Res 1999, 52:137-144.

58. Omogbenigun FO, Nyachoti CM, Slominski BA: Dietary supplementation with multienzyme preparations improved nutrient utilization and growth performance in weaned pigs. J Anim Sci 2004, 82:1053-1061

59. Yin YL, McEvoy JDG, Schulze H, Henning U, Souffrant WB, McCracken K: Apparent digestibility (ileal and overall) of nutrients and endogenous nitrogen losses in growing pigs fed wheat (var. Soissons) or its byproducts without or with xylanase supplementation. Livest Prod Sci 2000 62:119-132.

60. Moeser AJ, van Kempen TATG: Dietary fibre level and enzyme inclusion affects nutrient digestibility and excreta characteristics in grower pigs. J Sci Food Agric 2002, 82:1606-1613.

61. Li S, Sauer WC, Mosenthin R, Kerr B: Effect of $\beta$-glucanase supplementation of cereal-based diets for starter pigs on the apparent digestibilities of dry matter, crude protein and energy. Anim Feed Sci Tech 1996, 59:223-231.

62. Pettey LA, Carter SD, Senne BW, Shriver JA: Effects of beta-mannanase addition to corn-soybean meal diets on growth performance, carcass traits, and nutrient digestibility of weanling and growing-finishing pigs. J Anim Sci 2002, 80:1012-1019.

63. Kim SW, Knabe DA, Hong KJ, Easter RA: Use of carbohydrases in corn-soybean meal-based nursery diets. J Anim Sci 2003, 81:2496-2504.

64. Ji F, Casper DP, Brown PK, Spangler DA, Haydon KD, Pettigrew JE: Effects of dietary supplementation of an enzyme blend on the ileal and fecal digestibility of nutrients in growing pigs. J Anim Sci 2008, 86:1533-1543.

65. Spencer JD, Petersen Gl, Gaines AM, Augspurger NR: Evaluation of different strategies for supplementing distiller/s dried grains with solubles (DDGS) to nursery pig diets. J Anim Sci 2007, 85(Suppl. 2):169.

66. Kerr BJ, Weber TE, Shurson GC: Evaluation of commercially available enzymes, probiotics, or yeast on apparent nutrient digestion and growth in nursery and finishing pigs fed diets containing corn-dried distillers grains with solubles. Prof Anim Sci; (submitted).

67. NRC: Nutrient Requirements of Swine. 9th edition. Washington, D.C: Natl. Acad. Press; 1998.

68. Adeola O, Sands JS, Simmins PH, Schulze H: The efficacy of an Escherichia coli-derived phytase preparation. J Anim Sci 2004, 82:2657-2666.

69. Adeola O, Olukosi OA, Jendza JA, Dilger RN, Bedford MR: Response of growing pigs to Peniophora lycii- and Escherichia coli-derived phytases or varying ratios of calcium to total phosphorus. Anim Sci 2006, 82:637-644.

70. Liao SF, Kies AK, Sauer WC, Zhang YC, Cervantes M, He JM: Effect of phytase supplementation to a low- and a high-phytate diet for growing pigs on the digestibilities of crude protein, amino acids, and energy. J Anim Sci 2005, 83:2130-2136.

71. Jendza JA, Dilger RN, Sands JS, Adeola O: Efficacy and equivalency of an Escherichia coli-derived phytase for replacing inorganic phosphorus in the diets of broiler chickens and young pigs. J Anim Sci 2006, 84:3364-3374.

72. Beaulieu AD, Bedford MR, Patience JF: Supplementing corn or corn-barley diets with an E. coli derived phytase decreases total and soluble P output by weanling and growing pigs. Can J Anim Sci 2007, 87:353-364.

73. Brady SM, Callan JJ, Cowan D, McGrane M, O/Doherty JV: Effect of phytase inclusion and calcium/phosphorus ratio on the performance and nutrient retention of grower-finisher pigs fed barley/wheat/soya bean meal-based diets. J Sci Food Agric 2002, 82:1780-1790.

74. Shelton JL, Southern LL, Bidner TD, Persica MA, Braun J, Cousins B, McKnight F: Effect of microbial phytase on energy availability and lipid and protein deposition in growing swine. J Anim Sci 2003, 81:2503-2062

75. Jendza JA, Dilger RN, Adedokun SA, Sands JS, Adeola O: Escherchia coli phytase improves growth performance of starter, grower, and finisher pigs fed phosphorus-deficient diets. J Anim Sci 2005, 83:1882-1889.

76. Veum TL, Dollinger DW, Buff CE, Bedford MR: A genetically engineered Escherichia coli phytase improved nutrient utilization, growth performance, and bone strength of young swine fed diets deficient in available phosphorus. J Anim Sci 2006, 84:1147-1158.

77. Kerr BJ, Weber TE, Miller PS, Southern LL: Effect of phytase on apparent total tract digestibility of phosphorus in corn-soybean meal diets fed to finishing pigs. J Anim Sci 2010, 88:238-247.

78. Olukosi OA, Sands JS, Adeola O: Supplementation of carbohydrases or phytase individually or in combination to diets for weanling and growing-finishing pigs. J Anim Sci 2007, 85:1702-1711.

79. Xu G, He G, Baidoo SK, Shurson GC: Effect of feeding diets containing corn distillers dried grains with solubles (DDGS), with or without phytase, on nutrient digestibility and excretion in nursery pigs. J Anim Sci 2006a, 84(Suppl. 2):122. Abstr.

80. Xu G, Whitney MH, Shurson GC: Effects of feeding diets containing corn distillers dried grains with solubles (DDGS), with or without phytase, on nutrient digestibility and excretion in grow-finish pigs. J Anim Sci 2006b, 84(Suppl. 2):123. Abstr.

81. Lindemann MD, Apgar GA, Cromwell GL, Simmins PH, Owusu-Asiedu A: Supplementation with phytase and xylanase can increase energy availability in swine diets containing corn distillers dried grains with solubles (DDGS). J Anim Sci 2009, 87(E-Suppl. 2):69. Abstr.

82. Stein HH, Shurson GC: The use and application of distillers dried grains with solubles in swine diets. J Anim Sci 2009, 87:1292-1303.

83. Pedersen C, Boersma MG, Stein HH: Digestibility of energy and phosphorus in ten samples of distillers dried grains with solubles fed to growing pigs. J Anim Sci 2007, 85:1168-1176. 
84. Anderson PV, Kerr BJ, Weber TE, Ziemer CJ, Shurson GC: Determination and prediction of energy from chemical analysis of corn co-products fed to finishing pigs. J Anim Sci 2012, 90:1242-1254.

85. Leathers TD: Upgrading fuel ethanol coproducts. SIM News. 1998, 48:210-217.

86. Urriola PE, Shurson GC, Stein HH: Digestibility of dietary fiber in distillers coproducts fed to growing pigs. J Anim Sci 2010, 88:2373-2381.

doi:10.1186/2049-1891-4-11

Cite this article as: Kerr and Shurson: Strategies to improve fiber utilization in swine. Journal of Animal Science and Biotechnology 2013 4:11.

\section{Submit your next manuscript to BioMed Central and take full advantage of:}

- Convenient online submission

- Thorough peer review

- No space constraints or color figure charges

- Immediate publication on acceptance

- Inclusion in PubMed, CAS, Scopus and Google Scholar

- Research which is freely available for redistribution 